\title{
Estrategias de afrontamiento, empatía y tendencia prosocial en universitarios
}

\author{
Francisco Manuel Morales \\ Universidad de Granada (España)
}

\begin{abstract}
El objetivo del presente estudio consistió en analizar las estrategias de afrontamiento del estrés cotidiano percibido en una muestra de estudiantes universitarios y su relación con otras variables psicoeducativas de empatía y medidas de tendencia prosocial. Los participantes fueron estudiantes universitarios pertenecientes a primeros cursos de las Facultades de Ciencias de la Educación y de Psicología a los que se les aplicó los siguientes instrumentos: un Inventario de Estrategias de Afrontamiento, Test de Empatía Cognitiva y Afectiva, una Escala de Prosocialidad y otro para evaluar Comportamientos Prosociales/ Medida de Tendencia Prosocial. Los resultados demuestran la existencia de correlaciones positivas entre estrategias productivas de afrontamiento centradas en el problema como la resolución de problemas y las variables de empatía, conducta prosocial y medida de tendencia prosocial. La estrategia evitación se asoció negativamente con la variable empatía y comportamientos prosociales. Los datos obtenidos son relevantes para el diseño de futuros programas de entrenamiento de estrategias de afrontamiento del estrés cotidiano en el ámbito universitario y para el desarrollo de competencias intrapersonales e interpersonales como las relacionadas con la empatía y el desarrollo de comportamientos prosociales.
\end{abstract}

Palabras clave: Estrategias de afrontamiento, estrés cotidiano, universitarios, empatía, conducta prosocial.

Coping strategies, empathy and prosocial tendencies in university students. The aim of this study was to analyze the coping strategies of daily stress perceived in a sample of university students and its relationship with other psycho-educational variables of empathy and measures of prosocial tendency. The participants were university students belonging to first years of the Education Sciences and Psychology Faculties to whom the following instruments were applied: an Inventory of Coping Strategies, Cognitive and Affective Empathy Test, a Prosocial Scale and another one to evaluate Prosocial Behaviors/Measurement of prosocial tendency. The results proof the existence of positive correlations between productive problem-centered coping strategies such as problem solving and the empathy, prosocial behavior variables, and prosocial trend measurement. The avoidance strategy was negatively associated with the empathy variable and prosocial behavior. The data obtained are relevant for the design of future training programs for coping with everyday stress in the university setting and for the development of intrapersonal and interpersonal skills such as those related to empathy and the development of prosocial behaviors.

Keywords: Coping strategies, daily stress, college students, empathy, prosocial behavior.

Correspondencia: Francisco Manuel Morales. Departamento de Psicología Evolutiva y de la Educación. Facultad de Psicología. Universidad de Granada. Campus Universitario de Cartuja, s/n, C.P. 18071. Granada (España). E-mail: fmmorales@ugr.es 
Existen pocas investigaciones en el ámbito universitario centradas en evaluar las estrategias de afrontamiento del estrés cotidiano y aún son menos los que analizan las relaciones del afrontamiento centrado en el problema y en las emociones con variables como empatía, tendencia prosocial y comportamientos prosociales en universitarios, como se hace en el presente estudio.

Aún hay falta de consenso sobre la operacionalización del constructo "coping", y sobre las categorías de afrontamiento que mejor discriminan (Compas, Connor-Smith, Saltzman, Thomsen, y Wadsworth, 2001). Teniendo en cuenta la complejidad del proceso de afrontamiento, un marco teórico podría proporcionar una estructura para integrar el vasto cuerpo de investigación. Skinner, Edge, Altman, y Sherwood (2003) han propuesto una tipología para clasificar las formas de hacer frente. Distinguieron las categorías unidimensionales de orden inferior de las categorías multidimensionales de orden superior y las familias de afrontamiento. De los más de 1.000 diferentes sistemas de clasificación de afrontamiento revisados por Skinner et al. (2003), solo cuatro sistemas de categorías fueron construidos empíricamente, basados en la teoría y sometidos a técnicas estadísticas confirmatorias con las cuales los ajustes de maneras más bajas de enfrentarse a categorías de orden superior fueron probados.

Una de las definiciones ampliamente aceptadas sobre el término afrontamiento fue la propuesta por Lazarus (1966), que lo entienden como un proceso que se activa cuando se percibe una amenaza, con la finalidad de contribuir a la regulación del conflicto emocional y a la eliminación de la amenaza. Posteriormente, Lazarus y Folkman (1984) consideraron que el afrontamiento constituye la respuesta apropiada al estrés, la cual describen como "esfuerzos cognitivos y comportamentales, constantemente cambiantes, para manejar las demandas específicas externas o internas apreciadas como excedentes o que desbordan los recursos del individuo" (p. 164). Este enfoque inicial es aceptado por teóricos actuales del afrontamiento (García, 2010; Griffith, Dubow, e Ippolito, 2000).

Las estrategias de afrontamiento son entendidas como esfuerzos conscientes y voluntarios para regular emociones, conductas, cogniciones, psicofisiología y variables del entorno en respuesta al estrés de acontecimientos diarios o circunstancias (Compas et al., 2001; Frydenberg y Lewis, 1993; Trianes, 2002). Estos recursos implican dos dimensiones de la persona: a) la interna, relativa a variables disposicionales, herencia, edad y sexo, y b) la externa, relacionada con acciones ante demandas de la situación específica o el entorno sociocultural. Ambas dimensiones interactúan predisponiendo a las personas a comportamientos concretos y característicos (Zeidner y Saklofske, 1996).

Las estrategias de afrontamiento se definen como el proceso dinámico de intentar reducir o eliminar los estresores a través de respuestas cognitivas o de comportamiento (Lazarus y Folkman, 1984). La estrategia de afrontamiento utilizada puede reducir o exacerbar los sentimientos negativos que resultan de un evento 
estresante y / o puede ayudar a eliminar los efectos del evento estresante todos juntos. El ajuste que sigue a una situación estresante se ve como una función de las estrategias de enfrentamiento implementadas. El paso de la evaluación secundaria (Lazarus, 1991) sugiere que los individuos, dada una situación estresante, evaluarán su capacidad para reducir el impacto de las emociones negativas provocadas por el evento. Es en este proceso que los individuos perciben si tienen o no los recursos adecuados para manejar una situación estresante. Si se cree que estos recursos son bajos, cualquier emoción negativa aparente es exacerbada (Lengua y Long, 2002). Si se cree que los recursos son altos y el individuo se involucra en una estrategia de afrontamiento apropiada, el nivel de estrés a menudo se minimiza o elimina (Lazarus, 1991; Lengua y Long, 2002).

Se espera encontrar relaciones entre las estrategias de afrontamiento empleadas y el grado de ajuste, adaptación o psicopatología (Compas et al., 2001). Connor-Smith y Compas (2002) encontraron que varias estrategias de afrontamiento (por ejemplo, el compromiso de control primario, el compromiso de control secundario y el afrontamiento de la separación) moderaron las relaciones entre la reactividad al estrés involuntaria, el estado de salud y los problemas de internalización. Las reacciones efectivas / ajustadas fueron aquellas reacciones, que proporcionaron un efecto de amortiguación, mientras que las reacciones de afrontamiento ineficaces / inadaptadas ponen al individuo en riesgo de desarrollar problemas mentales. Compas et al. (2001) revisaron los estudios empíricos de la asociación entre el afrontamiento y los resultados psicológicos. Llegaron a la conclusión de que el afrontamiento del compromiso/aproximación y centrado en el problema se asociaron con un mejor ajuste, y que el afrontamiento de la separación y el afrontamiento centrado en la emoción sin una adecuada expresión y regulación de la misma se asociaron con un ajuste psicológico más deficiente. Sin embargo, estos hallazgos generales no fueron concluyentes y la calidad del ajuste pareció estar relacionada con la naturaleza del estresante o bien controlable o no.

Respecto a los tipos de estrategias de afrontamiento, Lazarus y Folkman (1984) diferencian entre estrategias de afrontamiento centradas en el problema y estrategias de afrontamiento centradas en reducir la emoción. En el modelo aceptado de otros investigadores como Frydenberg y Lewis (1996), se diferencia entre tres estilos básicos de afrontamiento: a) centrado en el problema (concentrarse en resolver el problema, esforzarse y tener éxito, fijarse en lo positivo, diversiones relajantes y distracción física); b)en relación a los demás (apoyo social, hacer amigos íntimos, buscar pertenencia, acción social, apoyo espiritual y buscar apoyo profesional); y c) estilo improductivo (preocuparse excesivamente, hacerse ilusiones, no afrontar el problema, reducción de tensión, ignorar el problema, autoinculparse y reservarlo para sí) (p. x). 
Un mayor nivel de empatía o predisposición del grupo para una convivencia entre estudiantes con más comprensión y expresión adecuada de las emociones (Sierra et al., 2015) y al mismo tiempo una mejor comunicación interpersonal y de la mejora de la convivencia favorece entornos educativos de inclusión y con adecuados canales para la comunicación ayuda a la prevención del estrés (Martínez-Otero, 2011; Sierra et al., 2015) y ello apuntaría a un mejor rendimiento académico (Sierra et al., 2015).

En algún estudio previo (Morales, 2017) se demostró que las estrategias productivas de afrontamiento, como solución activa, búsqueda de apoyo, y actitud positiva influyen en el autoconcepto académico, las habilidades sociales y la inteligencia emocional en sus distintas dimensiones.

La noción de expresión y regulación emocional efectiva, específicamente al examinar el papel del comportamiento prosocial como un mecanismo de afrontamiento, es un modelo que los investigadores han indicado que es apropiado (Eisenberg, Fabes, Karbon, Murphy, y Juhnke, 1996). La regulación emocional es el proceso afectivo, cognitivo y conductual a través del cual un individuo influye tanto en su experiencia como en su expresión emocional (Gross, 1998). Desafortunadamente, como lo señalan Goodman y Sotham-Gerow (2010), una limitación clave en la literatura de victimización por pares ha sido el fracaso en examinar el papel de las emociones en el enfrentamiento con la victimización. La investigación actual sobre la victimización puede beneficiarse del examen de la regulación emocional porque las variaciones en la regulación emocional y la respuesta pueden, en parte, explicar las diferencias en el ajuste, por ejemplo, a la victimización por pares. El conocimiento de la regulación emocional es, por lo tanto, potencialmente importante al examinar la heterogeneidad en las estrategias de afrontamiento de los individuos victimizados y al sugerir por qué algunas personas enfrentan comportamientos prosociales y otras no.

La respuesta emocional negativa o la falta de regulación emocional puede conducir a un enfrentamiento ineficaz y un mayor desajuste como la depresión y otros problemas de conducta (Sandler et al., 2000). La falta de regulación emocional también se asocia con niveles más altos de comportamientos sociales negativos hacia compañeros (por ejemplo, agresión, Eisenberg et al., 1993). Aunque significativamente menos estudios han examinado las respuestas emocionales positivas y el enfrentamiento en situaciones estresantes, las que se han reportado revelan niveles bajos de problemas de internalización (Jackson y Warren, 2000). Parece que la capacidad de auto-regulación puede disminuir los efectos del estrés en el ajuste psicológico (Lengua y Long, 2002). Asimismo, la autorregulación también es predictiva de la respuesta activa (enfoque), ayudando en la mitigación del impacto de los eventos negativos de la vida en el ajuste del individuo. Además, los hallazgos anteriores han indicado una valoración positiva de una situación estresante (la creencia de que uno tiene la capacidad de lidiar con la situación) es a menudo predictivo del afrontamiento activo (Jackson y Warren, 2000). 
El compromiso en los comportamientos prosociales a menudo aumenta en la última infancia y en la adolescencia temprana y se asocia con la maduración de la capacidad cognitiva para tomar la perspectiva de otra persona. Hoffman (1982) sugiere que el aumento de la capacidad de toma de perspectiva da lugar a la capacidad de diferenciar entre el propio sufrimiento y el de otro, una capacidad necesaria para una adecuada regulación emocional. La toma de perspectiva permite desarrollar una sensación de simpatía por los demás y su desarrollo se correlaciona a menudo con la regulación emocional y el comportamiento prosocial (Carlo, Hausmann, Christiansen, y Randall, 2003). Hoglund (2005) también encontró que las diferencias individuales son evidentes en el nivel de capacidades social-cognitivas (toma de perspectiva y habilidades interpersonales).

La investigación desarrollista conceptualizó la relación entre comportamiento agresivo y prosocial como dos polos del mismo constructo conductual, argumentando que las respectivas variables subyacentes representan extremos opuestos de un constructo más amplio basado en la evidencia de que el comportamiento prosocial es un comportamiento positivo y agresivo está negativamente relacionado con las terceras variables comunes, tales como la empatía (Eisenberg y Miller, 1987). Los dos comportamientos son constructos conceptualmente relacionados, y numerosas teorías de desarrollo, como la teoría de la socialización (Hastings, Utendale, y Sullivan, 2007) han elaborado que ambos comportamientos son el resultado de mecanismos causales similares.

La conducta prosocial define cualquier forma de comportamiento realizado voluntariamente con el propósito de producir un beneficio para otras personas (Eisenberg, 2003; Eisenberg, Fabes, y Spinrad, 2006). Este término es equivalente al término solidaridad, utilizado con más frecuencia el ámbito de la sociología (Lindenberg, 2006). Existe un consenso cada vez mayor de que la comprensión del comportamiento prosocial require un enfoque multidimensional que considere la variedad de mecanismos distintos que pueden conducir a diferentes respuestas prosociales (Paulus, 2014). Según el mismo, el comportamiento prosocial no es un constructo unidimensional, sino que incluye una amplia variedad de conductas (Padilla-Walker y Carlo, 2014), entre las que se encuentran la ayuda a los demás, el altruismo, el voluntariado o la cooperación como las que más frecuentemente han sido estudiadas (Schroeder y Graziano, 2015).

Puede señalarse que los comportamientos prosociales pueden vincularse con otras habilidades y comportamientos no cognitivos, como la autoeficacia (Caprara y Steca, 2005), la autoestima (Afolabi, 2014), el afrontamiento (de Boo y Wicherts, 2009) y la autorregulación (Blake, Piovesan, Montinari, Warneken, y Gino, 2015). En la mayoría de los casos, los estudios que examinan comportamientos antisociales o prosociales también han examinado la competencia social, la aceptación o rechazo de 
pares, la presencia de estresores, y los problemas psicológicos, como depresión, ansiedad, hiperactividad y problemas de atención. No obstante, apenas existen estudios centrados en las relaciones con estrategias de afrontamiento del estrés cotidiano en universitarios y las variables psicoeducativas indicadas de empatía y conducta prosocial.

Un importante tema de estudio en el área de la educación y el desarrollo en jóvenes y adolescentes es la relación que existe entre las estrategias productivas de afrontamiento, conducta prosocial y el rendimiento académico. La investigación sobre los resultados educativos ha demostrado la importancia de comportamientos sociales positivos en la promoción del rendimiento académico. Los programas educativos que se centran en el desarrollo de las competencias sociales han ganado popularidad como un medio para crear un ambiente positivo para el aprendizaje, incrementar las calificaciones de los estudiantes y mejorar sus habilidades socioemocionales (Catalano, Berglund, Ryan, Lonczak, y Hawkins, 2004).

Precisamente, el objetivo del presente estudio fue evaluar las estrategias de afrontamiento del estrés cotidiano percibido en una muestra de estudiantes universitarios y su relación con otras variables psicoeducativas de empatía, prosocialidad y medidas de tendencia prosocial. Se esperaba encontrar la existencia de relaciones positivas entre el empleo de estrategias de afrontamiento centradas en el problema y la puntuación en la variable empatía y un mayor número de comportamientos prosociales en coherencia con alguna investigación previa centrada especialmente en la etapa evolutiva de la adolescencia. En universitarios aún se localizan pocos estudios centrados en analizar las relaciones entre dichos constructos.

\section{MÉTODO}

\section{Participantes}

Los participantes fueron 200 estudiantes universitarios pertenecientes a primeros cursos de las Facultades de Ciencias de la Educación y de Psicología, la mayoría mujeres, con edades entre los 18 y 26 años. Se trabajó con una muestra incidental equilibrada según el género. Se excluyó al estudiantado con el reconocimiento de la condición a tiempo parcial y con Necesidades Educativas Especiales.

\section{Instrumentos}

Inventario de Estrategias de Afrontamiento (Tobin, Holroyd, Reynolds, y Kigal, 1989. Adaptación por Cano, Rodríguez, y García, 2006). Permite evaluar el uso de determinadas estrategias de afrontamiento la situación estresante según escala tipo Likert de cinco escalones siendo 0 "En absoluto" y 4 "Totalmente". Un ejemplo de ítem del instrumento es "Me culpé a mí mismo" o "Pasé algún tiempo solo". Este instrumento 
presenta adecuadas propiedades psicométricas de fiabilidad y validez. La consistencia interna para cada una de las estrategias oscila entre 0.53 y 0.66 .

Medida de Tendencia Prosocial Revisado (PTM-R) (Rodríguez, 2017; Mestre, Carlo, Samper, Tur-Porcar, y Mestre, 2015). Consta de 21 ítems según escala de cinco puntos representando el 1 No me describe bien y el 5 Me describe muy bien y examina la estructura y funciones de una medida multidimensional de conductas prosociales. Incluye el análisis de las relaciones entre formas específicas de comportamientos prosociales y la simpatía, la toma de perspectiva, y la medida global del comportamiento prosocial. Evalúa 6 tipos de comportamiento prosocial: público (intención de beneficiar a otros en presencia de los demás), emocional (intención de beneficiar a otros, pero bajo circunstancias emocionalmente emotivas), emergencia (ayuda bajo situaciones de crisis), altruista (ayuda sin esperar nada a cambio), anónimo (ayuda sin la mirada de nadie) y complacencia u obediencia (ayuda cuando los demás lo solicitan). Los ítems usados son por ejemplo "Prefiero dar dinero sin que nadie lo sepa" o "Cuando otros se sienten muy tristes, normalmente les ayudo", medidos mediante puntuaciones del 1 al 5. La consistencia interna para la simpatía es 0.67, para la toma de perspectiva es 0.71 y para la puntuación global es de 0.78. Este método se ha aplicado con anterioridad en estudios como el de Carlo et al. (2003) con el objetivo de medir las tendencias prosociales.

Escala de Prosocialidad de Caprara, Steca, Zelli, y Capanna (2005); (Rodríguez et al., 2017). Está formada por 16 ítems autoinformados según escala del 1 al 5 , representando el uno el valor Nunca/casi nunca y el 5 el valor Siempre/casi siempre. Evalúa las diferencias individuales en cuanto a la prosociabilidad en la población adulta. Un ejemplo de ítem es: "Intento ayudar a los demás" una escala del 1 al 5, representando el uno el valor Nunca/casi nunca y el 5 el valor Siempre/casi siempre. Engloba un conjunto de acciones voluntarias que una persona puede adoptar para ayudar, cuidar, asistir o confortar a otros. La escala presenta una consistencia interna de 0.84 , medido mediante alfa de Cronbach.

Test de Empatía Cognitiva y Afectiva (TECA, López-Pérez, Fernández-Pinto, y García, 2008). Este instrumento consta de 33 ítems según escala Likert con unos valores que oscilan entre 1 (Totalmente en desacuerdo) y 5 (Totalmente de acuerdo). Incluye la dimensión afectiva y cognitiva de la empatía: comprensión emocional, adopción de perspectivas, estrés empático y alegría empática. Un ejemplo de ítem de este instrumento es: "Me cuesta llorar con lo que les sucede a otros/as". Este instrumento presenta adecuadas propiedades psicométricas de fiabilidad y validez. La escala presenta una consistencia interna de 0.86 . 


\section{Procedimiento}

Los cuestionarios se aplicaron de forma colectiva en el grupo clase asegurando el anonimato, la confidencialidad de la información, la posibilidad de abandonar el estudio en cualquier momento sin dar ningún tipo de explicación, así como también se recogió el consentimiento informado de los participantes en el estudio. El tiempo medio para completar los cuestionarios fue de unos 14 minutos.

\section{Análisis de datos}

Se utilizó un diseño ex post facto. El estudio fue aprobado por el Comité de Ética en Investigación de la Universidad de Granada. Se realizaron análisis descriptivos y de correlación de Pearson entre las variables.

\section{RESULTADOS}

A continuación, en la tabla 1 se muestran los estadísticos descriptivos del presente estudio.

Tabla 1. Descriptivos de estrategias de afrontamiento del estrés cotidiano, prosocialidad, empatía $\mathrm{y}$ medidas de tendencia prosocial en estudiantes univesitarios

\begin{tabular}{|c|c|c|c|c|}
\hline Variables & Min & Max & Media & $D T$ \\
\hline Prosocialidad & 28.00 & 77.00 & 64.33 & 8.19 \\
\hline Conducta prosocial & 11.00 & 30.00 & 24.77 & 3.45 \\
\hline Empatía y apoyo emocional & 7.00 & 20.00 & 15.79 & 2.53 \\
\hline \multicolumn{5}{|l|}{ Empatía } \\
\hline Adopción de perspectivas & 19.00 & 40.00 & 30.15 & 3.88 \\
\hline Comprensión emocional & 25.00 & 49.00 & 36.32 & 5.07 \\
\hline Estrés empático & 17.00 & 40.00 & 28.21 & 4.12 \\
\hline Alegría empática & 21.00 & 35.00 & 29.80 & 3.58 \\
\hline \multicolumn{5}{|l|}{ Medida de tendencia prosocial } \\
\hline Público & 3.00 & 15.00 & 5.89 & 3.01 \\
\hline Emocional & 7.00 & 25.00 & 18.72 & 3.77 \\
\hline Emergencia & 5.00 & 15.00 & 11.46 & 2.06 \\
\hline Anónimo & 4.00 & 20.00 & 11.65 & 4.22 \\
\hline Altruista & 5.00 & 20.00 & 16.99 & 3.26 \\
\hline Complacencia u obediencia & 4.00 & 10.00 & 8.38 & 1.40 \\
\hline \multicolumn{5}{|l|}{ Estrategias de afrontamiento } \\
\hline Resolución de problemas & 0.00 & 20.00 & 11.68 & 5.33 \\
\hline Autocrítica & 0.00 & 20.00 & 7.26 & 6.49 \\
\hline Expresión emocional & 0.00 & 24.00 & 14.56 & 6.61 \\
\hline Pensamiento desiderativo & 0.00 & 16.00 & 11.36 & 4.58 \\
\hline Reestructuración cognitiva & 1.00 & 20.00 & 10.67 & 5.36 \\
\hline Evitación de problemas & 0.00 & 16.00 & 6.17 & 3.52 \\
\hline Retirada social & 3.00 & 16.00 & 10.36 & 2.49 \\
\hline
\end{tabular}

$D T=$ Desviación típica.

En la tabla 2 se presentan los resultados del análisis correlacionan entre las variables. En términos generales, se encuentran correlaciones entre las estrategias de afrontamiento del estrés cotidiano empleadas por el estudiantado universitario y las 
variables prosocialidad, empatía y medidas de tendencia prosocial.

Se encontraron correlaciones positivas estadísticamente significativas entre la estrategia de afrontamiento del estrés cotidiano de resolución de problemas con las variables de empatía y apoyo emocional, la dimensión cognitiva de la variable empatía (adopción de perspectivas) y con la medida de tendencia prosocial de complacencia u obediencia.

Tabla 2. Correlaciones entre las variables estrategias de afrontamiento del estrés cotidiano, prosocialidad, empatía y medidas de tendencia prosocial en estudiantes universitarios

\begin{tabular}{|c|c|c|c|c|c|c|c|}
\hline Variables & REP & AUC & EXE & PSD & REC & EVP & RES \\
\hline \multicolumn{8}{|l|}{ Prosocialidad } \\
\hline $\mathrm{CP}$ & .10 & -.10 & $.24 *$ & -.04 & .03 & -.08 & -.02 \\
\hline EAE & $.25^{* *}$ & -.03 & $.33^{* *}$ & -.03 & .16 & -.04 & .07 \\
\hline \multicolumn{8}{|l|}{ Empatía: } \\
\hline $\mathrm{AP}$ & $.20 *$ & -.07 & $.28^{* *}$ & .01 & .09 & $-.20 *$ & .11 \\
\hline $\mathrm{CE}$ & .19 & -.09 & $.21^{* *}$ & -.11 & .14 & -.04 & -.05 \\
\hline $\mathrm{EE}$ & .15 & -.16 & $.26^{* *}$ & -.04 & .15 & -.03 & .04 \\
\hline $\mathrm{AE}$ & .18 & -.12 & .19 & .07 & .05 & -.19 & .09 \\
\hline \multicolumn{8}{|l|}{ : PTM-R } \\
\hline Público & .05 & $.27 * *$ & -.01 & -.17 & .17 & $.27 * *$ & -.17 \\
\hline Emocional & .18 & .08 & $.26^{* *}$ & .07 & .16 & .08 & .11 \\
\hline Emergencia & .11 & .06 & .18 & .00 & .14 & .06 & .06 \\
\hline Anónimo & -.04 & $.24^{*}$ & -.18 & -.05 & -.02 & $.25 * *$ & -.03 \\
\hline Altruista & -.04 & $-.33 * *$ & .10 & -.03 & -.19 & $-.33 * *$ & $.30^{* *}$ \\
\hline $\mathrm{CO}$ & $.23 *$ & -.03 & $.29 * *$ & .03 & -.14 & -.03 & .03 \\
\hline
\end{tabular}

La estrategia de afrontamiento autocrítica muestra correlación positiva con la medida de comportamiento prosocial público.

La estrategia expresión emocional muestra correlación con la variable prosocialidad (conducta prosocial y la empatía y apoyo emocional), la adopción de perspectivas, la comprensión emocional, el estrés empático, el comportamiento prosocial emocional y complacencia u obediencia.

La estrategia de afrontamiento de pensamiento desiderativo y reestructuración cognitiva no demuestran correlación en esta muestra con las variables prosocialidad, empatía y medida de tendencia prosocial.

La evitación de problemas se asoció negativamente con la dimensión cognitiva de la variable empatía en la subescala adopción de perspectiva. También se encuentran asociaciones estadísticamente significativas con tres de las medidas de tendencia prosocial (público, anónimo y altruista).

La estrategia de afrontamiento retirada social demuestra correlaciones positivas con la medida de tendencia de comportamiento prosocial altruista. 


\section{DISCUSIÓN Y CONCLUSIONES}

El objetivo del presente estudio fue examinar las estrategias de afrontamiento del estrés cotidiano percibido y la puntuación en las variables de empatía, prosocialidad y medidas de tendencia prosocial, así como las relaciones entre dichas variables en una muestra de estudiantes universitarios. En ese sentido, puede señalarse que aún existen pocos estudios centrados específicamente, como se hace en este estudio, que aporten evidencias empíricas en el análisis de las estrategias de afrontamiento del estrés codidiano en universitarios y sus relaciones con la conducta prosocial, empatía y regulación emocional a pesar de las influencias que pueden tener en el proceso de enseñanza/aprendizaje como en la percepción de la calidad de vida, bienestar y en el rendimiento académico.

Los resultados exhiben la existencia de relaciones positivas entre las estrategias consideradas a priori como estrategias productivas de afrontamiento de resolución de problemas con las variables de empatía, prosocialidad y medidas de tendencia prosocial. Otro estudio (Quintana, Montgomery, y Malaver, 2009) en el que se analizó las relaciones entre la conducta resiliente en espectadores de violencia entre pares en ambientes educativos y los modos de afrontamiento encontró que el espectador prosocial ante la violencia entre pares es el que recurrió con más frecuencia a estrategias de afrontamiento activo orientada a la solución del problema, planificación y la búsqueda de apoyo social. En la misma línea, en la investigación previa realizada por Samper, Mestre, Tur, Santolaria, y Llorca (2012) se analizaron las estrategias de afrontamiento y la conducta prosocial identificándose un tipo de afrontamiento más productivo y centrado en el problema en aquellas personas con mayor puntuación en prosocialidad.

Los resultados demuestran correlaciones inversas entre la estrategia de afrontamiento de evitación del problema que se viene considerando como menos productiva o funcional en términos generales con la variable de empatía y con la variable de comportamiento prosocial altruista. Otras investigaciones exhiben que las estrategias de tipo evitativo se asocian con síntomas depresivos, mayores niveles de comportamiento agresivo (en lugar de comportamientos prosociales) y ansiedad autoinformada (Seiffge-Krenke y Stemmler, 2002).

Estrategias como la expresión y regulación emocional se asocian a mayores niveles de empatía y comportamientos prosociales. Ello es coherente con otros trabajos en los que se indica que la capacidad de controlar las emociones negativas y de hacer frente de manera efectiva a situaciones estresantes y emocionales es integral en el desarrollo de comportamientos prosociales (Fabes y Eisenberg, 1992).

Se considera de mucho interés este tipo de análisis que permitan seguir profundizando si el estudiantado universitario que emplea determinados tipos de 
estrategias de afrontamiento del estrés cotidiano y la adversidad exhiben comportamientos más prosociales, conductas solidarias y de trabajo a favor de otros y mayores niveles de empatía.

Entre las limitaciones del presente estudio pueden señalarse la aplicación de medidas de autoinformes con el carácter subjetivo que ello conlleva y el hecho de que no puede hablarse de relaciones causales siendo además necesario considerar otro tipo de variables psicoeducativas que ayuden a explicar e intentar asumir modelos multivariados más robustos con una vinculación aún más precisa con determinados modelos teóricos.

No obstante, puede concluirse señalando que los datos obtenidos resultan de utilidad para el diseño de programas de intervención psicoeducativa que contribuyan a la mejora de la calidad de vida, al bienestar psicológico y a la prevención del estrés cotidiano generado por las situaciones académicas que tienen que afrontar para un entrenamiento de estrategias de afrontamiento más productivas o eficaces que además son especialmente relevantes y pueden actuar como factores protectores en la actual situación de pandemia generada por el COVID-19.

\section{REFERENCIAS}

Afolabi, O.A. (2014). Do Self Esteem and Family Relations Predict Prosocial Behaviour and Social Adjustment of Fresh Students? Higher Education of Social Science, 7(1), 26-34.

Blake, P.R., Piovesan, M., Montinari, N., Warneken, F., y Gino, F. (2015). Prosocial norms in the classroom: The role of self-regulation in following norms of giving. Journal of Economic Behavior y Organization, 115(July), 18-29.

Cano, F., Rodríguez, L., y García, J. (2007). Adaptación española del Inventario de Estrategias de Afrontamiento. Actas Esp Psiquiatr, 35(1), 29-39.

Caprara, G.V, Steca, P., Zelli, A., y Capanna, C. (2005). A new scale for measuring adults' prosocialness. European Journal and Psychology Assess, 21(2), 77-89.

Caprara, G.V., y Steca, P. (2005). Self-Efficacy Beliefs as Determinants of Prosocial Behavior Conducive to Life Satisfaction Across Ages. Journal of Social and Clinical Psychology, 24(2), 191-217.

Carlo, G., Hausmann, A., Christiansen, S., y Randall B.A. (2003). Sociocognitive and behavioral correlates of a measure of prosocial tendencies for adolescents. The Journal of Early Adolescence, 23(1), 107-134. doi:10.1177/0272431602239132

Catalano, R.F., Berglund, M.L., Ryan, J.A., Lonczak, H.S., y Hawkins, J.D. (2004). Positive youth development in the United States: research findings on evaluations of positive youth development programs. The ANNALS of the American Academy of Political and Social Science, 591(1), 98-124.

Compas, B.E., Connor-Smith, J.K., Saltzman, H., Thomsen, A.H., y Wadsworth, M.E. (2001). Coping with stress during childhood and adolescence: Problems, progress, and potential in theory and research. Psychological Bulletin, 127, 87-127.

Connor-Smith, J.K., y Compas, B.E. (2002). Vulnerability to social stress: Coping as a mediator or moderator of sociotropy and symptoms of anxiety and depression. Cognitive Therapy and Research, 26, 39-55. 
De Boo, G.M., y Wicherts, J.M. (2009). Assessing Cognitive and Behavioral Coping Strategies in Children. Cognitive Therapy and Research, 33, 1-20.

Eisenberg, N. (2003). Prosocial behavior, empathy, and sympathy. En M.H. Bornstein, L. Davidson, C.L.M. Keyes, y K.A. Moore (Eds.), Well-being: Positive development across the life course (pp. 253-265). Mahwah, NJ: Erlbaum.

Eisenberg, N., y Miller, P.A. (1987). The relation of empathy to prosocial and related behaviours. Psychological Bulletin, 101(1), 91-119.

Eisenberg, N., Fabes, R.A., Bernzweig, J., Karbon, M., Poulin, R., y Hanish, L. (1993). The relations of emotionality and regulation to preschoolers' social skills and sociometric status. Child Development, 64, 1418-1438.

Eisenberg, N., Fabes, R.A., Karbon, M., Murphy, B., y Juhnke, C. (1996). The relations of dispositional prosocial behavior to emotionality, regulation, and social functioning. Child Development, 67, 974-992.

Eisenberg, N., Fabes, R.A., y Spinrad, T. (2006). Prosocial development. En W. Damon, N. Eisenberg, y R. Lerner (Eds.), Handbook of child psychology: Social, emotional, and personality development. Vol. 3 (pp. 646-718). New York: Wiley.

Fabes, R.A., y Eisenberg, N. (1992). Young children's coping with interpersonal anger. Child Development, 63, 116-128.

Frydenberg, E., y Lewis, R. (1993). The Adolescent Coping Scale: Practitioners Manual. Melbourne: Australian Council for Educational Research.

Frydenberg, E., y Lewis, R. (1996). Escala de afrontamiento para adolescentes. Madrid: TEA.

Frydenberg, E., y Lewis, R. (1996). A replication study of the structure of the adolescent coping scale: Multiple forms and applications of a self-report inventory in a counseling and research context. European Journal of Psychological Assessment, 12, 224-235.

García, C. (2010). Conceptualization and measurement of coping during adolescence: A review of the Literature. Journal of Nursing Scholarship, 42, 166-185.

Goodman K.L., y Southam-Gerow, M.A. (2010). The regulating role of negative emotions in children's coping with peer rejection. Child Psychiatry Human Development, 41, 515-534.

Griffith, M.A., Dubow, E., e Ippolito, M.F. (2000). Developmental and cross-situational differences in adolescents' coping strategies. Journal of Youth and Adolescence, 29, 183-204.

Gross, J.J. (1998). The emerging field of emotion regulation: An integrative review. Review of General Psychology, 2, 271-299.

Hastings, P.D., Utendale, W.T., y Sullivan, C. (2007). The socialization of prosocial development. In Grusec J.E, and Hastings P.D, (Eds.), Handbook of Socialization: Theory and Research (pp. 638-664). New York, NY: Guilford Press.

Hoffman, M.L. (1982). Development of prosocial motivation: Empathy and guilt. In N. Eisenberg (Ed.), The development of prosocial behavior (pp. 281-338). New York: Academic Press.

Hoglund, W.L. (2005). Mediating effects of social-cognitive errors and skills for children experiencing peer relational, physical, and ethnic victimization. Dissertation Abstract International, 66(5-A), 1637.

Jackson, Y., y Warren, J. (2000). Appraisal, social support, and life-events: Predicting outcome behavior in school-age children. Child Development, 71, 1441-1457.

Lazarus, R.S. (1966). Psychological stress and the coping process. New York, NY: McGraw-Hill.

Lazarus, R.S. (1991). Emotion and adaptation. In Pervin, L. A. (Ed.). Handbook of personality: Theory and research (pp. 609-637). New York: Guilford. 
Lazarus, R., y Folkman, S. (1984). Stress, appraisal, and coping. New York: Springer Publishing Company.

Lengua, L.J., y Long, A.C. (2002). The role of emotionality and self-regulation in the appraisalcoping process: Tests of direct and moderating effects. Journal of Applied Developmental Psychology, 23, 471-493.

Lindenberg, S. (2006). Prosocial Behavior, Solidarity, and Framing Processes. En D. Fetchenhauer, A. Flache, B. Buunk, y S. Lindenberg (Eds.), Solidarity and Prosocial Behavior: An Integration of Sociological and Psychological Perspectives (pp. 23-44). New York: Springer US.

López-Pérez, B., y Fernández-Pinto, I., y García, F.J. (2008). TECA: Test de Empatía Cognitiva y Afectiva; TEA ediciones.

Martínez-Otero, V. (2011). La empatía en la educación: estudio de una muestra de alumnos universitarios. Revista Electrónica de Psicología Iztacala, 14, 174-190.

Mestre, M.V., Carlo, G, Samper, P, Tur-Porcar, A.M., y Mestre, A.L. (2015). Psychometric Evidence of a Multidimensional Measure of Prosocial Behaviors for Spanish Adolescents. Journal Genetical and Psychology, 176(4), 260-71.

Morales, F.M. (2017). Relaciones entre afrontamiento del estrés cotidiano, autoconcepto, habilidades sociales e inteligencia emocional. European journal of education and psychology, 10, 41-48.

Padilla-Walker, L.M., y Carlo, G. (2014). The Study of Prosocial Behavior: Past, Present, and Future. In: L.M. Padilla-Walker, y G. Calvo (Eds.), Prosocial Development: A Multidimensional Approach (pp. 3-16). Oxford: Oxford University Press.

Paulus, M. (2014). The emergence of prosocial behavior: Why do infants and toddlers help, comfort, and share? Children Development Perspective, 8, 77-81. doi:10.1111/cdep.12066

Quintana, A., Montgomery, W., y Malaver, C. (2009). Modos de afrontamiento y conducta resiliente en adolescentes espectadores de violencia entre pares. Revista IIPSI, Faculta de Psicología UNMSM, 12(1), 153-171.

Rodríguez, LM. (2017). Adaptación de la Escala de Prosocialidad de Caprara en Adolescentes Argentinos. Revista Evaluar, 17(2), 177-187.

Samper, P., Mestre, V., Tur, A., Santolaria, A., y Llorca, A. (2012). Los mecanismos de afrontamiento como predictores de la disposición prosocial. Revista de Psicología y Educación, 7(2), 31-47.

Sandler, I.N., Kim, L.S., y MacKinnon, D. (2000). Coping and negative appraisal as mediators between control beliefs and psychological symptoms in children of divorce. Journal of Clinical Child Psychology, 29, 336-347.

Schroeder, D.A., y Graziano, W.G. (2015). The Field of Prosocial Behavior: An Introduction and Overview. In D.A. Schroeder, y W.G. Graziano (Eds.), The Oxford Handbook of Prosocial Behavior (pp. 3-36). Oxford: Oxford University Press.

Seiffge-Krenke, I., y Stemmler, M. (2002). Factors contributing to gender differences in depressive symptoms: A Test of three developmental models. Journal of Youth and Adolescence, 31, 405-417.

Sierra, L.O., Urrego, G., Montenegro, S., y Castillo, C. (2015). Estrés escolar y empatía en estudiantes de bachillerato practicantes de Mindfulness. Cuadernos De Lingüística Hispánica, 26, 175-197.

Skinner, E.A., Edge, K., Altman, J., y Sherwood, H. (2003). Searching for the structure of coping: A review and critique of category systems for classifying ways of coping. Psychological Bulletin, 129, 216-269.

Trianes, M.V. (2002). Estrés en la infancia. Madrid: Narcea. 
Zeidner, M., y Saklofske, D. (1996). Adaptive and maladaptive coping. En M. Zeidner, y N.E. Endler (Eds), Handbook of coping: theory, research, applications (pp. 234-365). Nueva York: Wiley.

Recibido: 29 de octubre de 2020

Recepción Modificaciones: 10 de noviembre de 2020

Aceptado: 18 de noviembre de 2020 